\title{
Local-field correction for an interstitial impurity in a crystal
}

\author{
Adel Rahmani, Patrick C. Chaumet, ${ }^{*}$ and Garnett W. Bryant \\ Atomic Physics Division, National Institute of Standards and Technology, Gaithersburg, Maryland 20899-8423
}

Received December 13, 2001

The local-field correction experienced by an interstitial impurity in a crystal with cubic symmetry is derived by use of a rigorous, self-consistent, semimicroscopic description of spontaneous emission in a microcavity. We compute the local-field factor for various positions of the impurity inside the crystal. Furthermore, we demonstrate that the local-field factor can be computed from a simple electrostatic model as a rapidly converging lattice sum. We show that the agreement between the predictions of this simple model and the rigorous calculations is remarkable, opening the way to a simple, general theory of a local-field effect for an impurity in a crystal with arbitrary symmetry. (C) 2002 Optical Society of America

OCIS codes: $260.2510,270.5580$.

The influence of the local environment on the electromagnetic properties of a nanosource is of crucial importance in modern optics and optoelectronics. As optical devices become smaller, the nature of the local-field correction experienced by a nanosource (atom, molecule, quantum dot) becomes a central issue. In this Letter we present a self-consistent calculation of the local-field correction experienced by an interstitial impurity source in a crystal with cubic symmetry. The local-field correction is obtained from a rigorous description of spontaneous emission ${ }^{1}$ that includes the Purcell effect and the dipole-dipole coupling between the source and the host.

Consider a two-level atom that is located at $\mathbf{r}_{0}$ in a microcavity and has an electric dipole transition moment along direction $\sigma=x, y, z$. The normalized decay rate is given by ${ }^{2,3}$

$$
\frac{\Gamma_{\sigma}}{\Gamma_{0}}=1+\frac{2}{2 k_{0}{ }^{3}} \operatorname{Im}\left[\overleftrightarrow{F}_{\sigma \sigma}\left(\mathbf{r}_{0}, \mathbf{r}_{0} ; \omega\right)\right]
$$

where $\Gamma_{0}$ and $k_{0}=\omega / c$ are the decay rate and the wave vector in free space, respectively, and $\overleftrightarrow{F}$ is the electric-field susceptibility that represents the electromagnetic response of the cavity. Finding the field susceptibility related to the expressions for the decay rate amounts to finding the electric field reflected back to the source by the cavity. To do so we use the coupled dipole method (CDM), which was developed for study of the scattering of light by particles with arbitrary shapes. ${ }^{4-12}$ In the CDM the scatterer is discretized over a cubic lattice. Details of the theory are given elsewhere. ${ }^{1,13,14}$ Local-field correction $L$ is defined as $^{13,14}$

$$
\Gamma=L^{2} \Gamma_{\text {cav }}+\delta \Gamma,
$$

where $\Gamma_{\text {cav }}$ represents the effect of the cavity geometry (reflection at the boundaries of the cavity). The term $\delta \Gamma$ represents the dissipative part of the dipole-dipole interaction between the source and the polarizable elements of the host medium (for a lossless medium, $\delta \Gamma=$ 0 ). When we consider a spherical cavity, we can use the theory of Chew ${ }^{15}$ to compute $\Gamma_{\text {cav }}$. By comparing this result and the CDM result, we can find $|L|$ from Eq. (2). ${ }^{13,14}$ Therefore the CDM allows us to compute rigorously the retarded, self-consistent local-field correction.

To gain a better insight into the rigorous scattering theory of the local-field correction it is useful to have a simple model to describe the local-field correction experienced by an impurity source in a crystal. Here we show how the local-field factor can be derived as a rapidly converging sum. In what follows, we consider an infinite crystal. However, it is implicit that all derivations are to be performed for a slab with finite thickness in the $z$ direction. The limit of an infinite crystal is taken once all the relevant lattice sums have been transformed into an absolutely convergent series by the planewise summation technique. ${ }^{17}$

Consider an infinite cubic lattice of point dipoles (polarizability $\alpha$ ) with lattice spacing $d$. We neglect retardation (electrostatic approximation) and assume that a uniform external field $\mathbf{E}^{\text {ext }}$ creates a uniform polarization $\mathbf{P}$ in the lattice. At any interstitial location in the lattice the local field is the sum of the external (applied) field and the field that is due to all the dipoles of the lattice (the case of a substitutional impurity is discussed in Refs. 13 and 14):

$$
\mathbf{E}^{\text {loc }}=\mathbf{E}^{\text {ext }}+\mathbf{E}^{\text {dip }} .
$$

Let $(a, b, c)$ be the coordinates, normalized by $d$, of the field point where we evaluate the local field inside a unit cell. We have $-1 / 2<x \leq 1 / 2$, where $x=a, b, c$ and $(a, b, c) \neq(0,0,0)$.

The total field created at an interstitial location $\mathbf{r}$ by the dipoles of the lattice is

$$
\mathbf{E}^{\operatorname{dip}}(\mathbf{r})=\sum_{i} \overleftrightarrow{F}_{0}\left(\mathbf{r}, \mathbf{r}_{i} ; 0\right) \frac{\alpha}{d^{3}} \mathbf{E}_{\mathrm{dip}}^{\mathrm{loc}}\left(\mathbf{r}_{i}\right),
$$

where the sum runs over the infinite lattice. $\quad \overleftrightarrow{F}_{0} / d^{3}$ is the vacuum field susceptibility. ${ }^{18}$ Because all dipoles experience the same local field, $\mathbf{E}_{\text {dip }}^{\text {loc }}$, we can write (the dependence on $\mathbf{r}$ is omitted henceforth)

$$
\mathbf{E}^{\text {dip }}=\frac{\alpha}{d^{3}} \overleftrightarrow{A} \mathbf{E}_{\text {dip }}^{\text {loc }}
$$

Tensor $\overleftrightarrow{A}$ contains all the lattice sums (sums over the lattice of the vacuum field susceptibility) for all dipole 
components. For instance, element $(i, j)$ of the tensor gives the lattice sum along direction $i$ that is due to a lattice of dipoles oriented along direction $j$. All the elements of $\overleftrightarrow{A}$ can be converted to rapidly converging sums that are readily computed by the general approach of De Wette and Schacher. ${ }^{19}$ Polarization $\mathbf{P}$ can be expressed as

$$
\mathbf{P}=\frac{\alpha}{d^{3}} \mathbf{E}_{\mathrm{dip}}^{\mathrm{loc}}=\frac{\epsilon-1}{4 \pi} \mathbf{E}^{\text {macro }},
$$

where $\mathbf{E}^{\text {macro }}$ is the macroscopic field. Using the continuity relations at an interface for the electric field and the electric induction, we also have

$$
\overleftrightarrow{\eta} \mathbf{E}^{\text {macro }}=\mathbf{E}^{\text {ext }}
$$

with

$$
\overleftrightarrow{\eta}=\left[\begin{array}{lll}
1 & 0 & 0 \\
0 & 1 & 0 \\
0 & 0 & \epsilon
\end{array}\right],
$$

where $\epsilon$ is the permittivity. This expression for $\overleftrightarrow{\eta}$ relates to our definition of the infinite crystal as the limit for infinite thickness of a slab normal to direction $z$. Equation (3) becomes

$$
\mathbf{E}^{\mathrm{loc}}=\overleftrightarrow{\eta} \mathbf{E}^{\text {macro }}+\frac{\epsilon-1}{4 \pi} \overleftrightarrow{A} \mathbf{E}^{\text {macro }}
$$

By definition, local-field factor $\overleftrightarrow{\Lambda}$ satisfies

$$
\mathbf{E}^{\text {loc }}=\overleftrightarrow{\Lambda} \mathbf{E}^{\text {macro }}
$$

Using Eqs. (9) and (10), we obtain

$$
\overleftrightarrow{\Lambda}=\frac{\epsilon-1}{4 \pi} \overleftrightarrow{A}+\overleftrightarrow{\eta}
$$

The local-field factor is obtained at once from Eq. (11). For example, the local field factor in direction $i$ is

$$
\left|L_{i}\right|=\left(\sum_{j=1}^{3}\left|\Lambda_{i j}\right|^{2}\right)^{1 / 2} .
$$

To foster understanding we first consider special high-symmetry cases. At certain locations of the lattice there is no mixing of polarization in the contribution of the dipoles to the local field, and the tensors $\overleftrightarrow{A}$ and $\overleftrightarrow{\Lambda}$ are diagonal. For example, an impurity source with a transition moment along $z$ and located at $(a, b, 0)$ or $(0,0, c)$ will experience a local-field correction

$$
L \equiv L_{z}=\frac{\epsilon-1}{4 \pi} A_{z z}+\epsilon
$$

with

$$
\begin{aligned}
A_{z z} & =\sum_{m=-\infty}^{\infty}\left[3(m+c)^{2} S_{5 / 2}-S_{3 / 2}\right], \\
S_{n} & =\sum_{k, l=-\infty}^{\infty}\left[(k+a)^{2}+(l+b)^{2}+(m+c)^{2}\right]^{-n} .
\end{aligned}
$$

Note that $A_{z z}$ is a geometrical factor that is independent of $\epsilon$. In Table 1 we list the values of dipole sum $A_{z z}$ and local-field factor $L_{z}$ at various locations inside the unit cell. The dipole sum satisfies ${ }^{17} A_{z z}(a, b, c)+$ $A_{z z}(c, a, b)+A_{z z}(b, c, a)=-8 \pi$.

Because the local-field factor in our CDM treatment of spontaneous emission is obtained from the decay rate (power losses) and not from the field, the CDM approach actually yields $|L|$. We plot in Figs. 1 and 2 the local-field factor found with the CDM (the direction of the transition moment of the source defines direction $z$ ). The dashed lines are the results computed from Table 1. The agreement is remarkable. Note that when $c=0$ (source in a lattice plane and oriented normally to it) $L=0$ for certain values of $\epsilon$, which would correspond to an inhibition of spontaneous emission by local-field effects. The reason for the excellent agreement between the rigorous CDM scattering theory and the simple quasi-static model is that the CDM calculation of the local field in a crystal is

Table 1. Dipole Sum and Local-Field Factor at Various Locations in the Lattice

\begin{tabular}{rrrcc}
\multicolumn{3}{c}{ Location } & & \\
\cline { 1 - 2 }$a$ & $b$ & $c$ & Dipole Sum $A_{z z}$ & Local-Field Factor $L_{z}$ \\
\hline $1 / 2$ & 0 & 0 & -23.4186008171 & $-0.864 \epsilon+1.864$ \\
$1 / 2$ & $1 / 2$ & 0 & -17.0453117498 & $-0.356 \epsilon+1.356$ \\
0 & 0 & $1 / 2$ & 21.7044604055 & $2.727 \epsilon-1.727$ \\
0 & $1 / 2$ & $1 / 2$ & -4.04371473946 & $0.678 \epsilon+0.322$ \\
$1 / 4$ & 0 & 0 & -73.5999217727 & $-4.857 \epsilon+5.857$ \\
0 & 0 & $1 / 4$ & 122.067102317 & $10.71 \epsilon-9.710$ \\
$1 / 2$ & $1 / 2$ & $1 / 2$ & -8.37758040957 & $(\epsilon+2) / 3$ \\
\hline
\end{tabular}

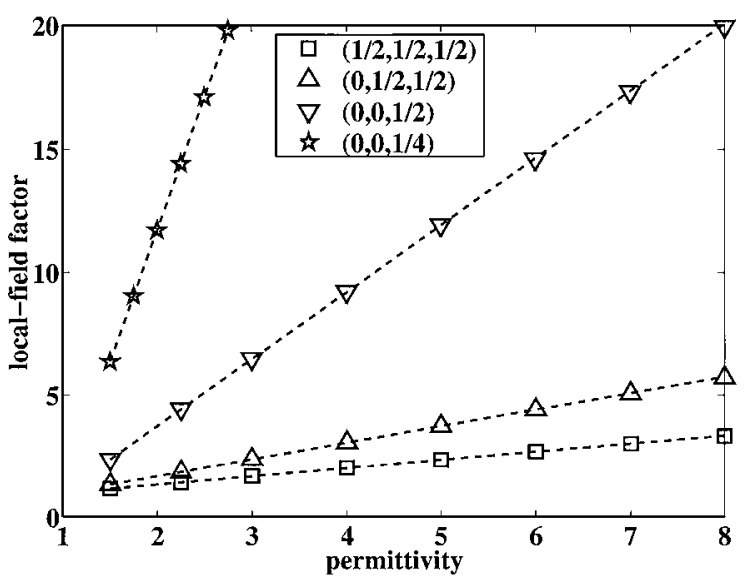

Fig. 1. Absolute value of the local-field factor versus permittivity $\epsilon$ for an interstitial source: symbols, self-consistent CDM calculation; dashed curves, results from Table 1 [Eq. (13)]. The numbers in parentheses give the $(a, b, c)$ coordinates of the source in the lattice. The transition moment of the source defines the $z$ direction. 


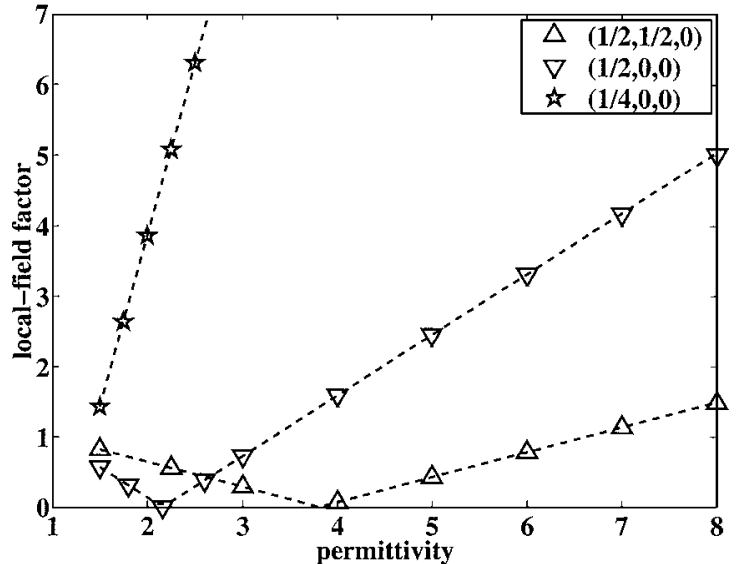

Fig. 2. Same as Fig. 1 but at different locations in the lattice.

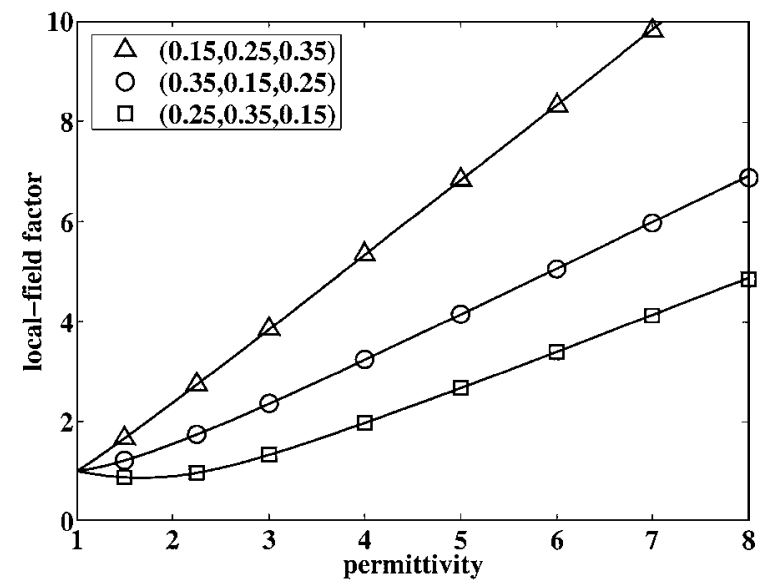

Fig. 3. Same as Fig. 1 but here we have computed the solid curves by using the generalized local-field factor [Eq. (12)].

meaningful only if $k_{0} d \ll 1$. When this condition is satisfied, because only the dipoles closest to the source make a significant contribution to the local-field, ${ }^{13,14}$ the phase associated with retardation remains small and has a negligible effect on the local-field correction.

Whereas in the special cases of Table 1 the local-field factor depends linearly on the permittivity, we emphasize that in the general case the linearity of the local-field factor with respect to the permittivity is only asymptotic (for large $\epsilon$ ). This condition is illustrated in Fig. 3, in which we compare Eq. (12) and our rigorous, self-consistent calculation for sources at some arbitrary location in the lattice. Again, the agreement is excellent.
In conclusion, we have used a rigorous, self-consistent scattering theory of spontaneous emission in microcavities to derive the local-field correction experienced by an interstitial impurity source in a crystal. We also presented a simple but general theory of localfield correction based on rapidly converging sums, whose validity we have demonstrated. Although we explicitly considered a cubic lattice here, this simple model of a local-field effect can be extended to crystals with lower symmetry by use of the appropriate lattice sums. ${ }^{19}$

Correspondence should be addressed to G. W. Bryant at garnett.bryant@nist.gov.

* Permanent address, Institut Fresnel (Unité Mixte de Recherche 6133), Faculté des Sciences et Techniques de St. Jérôme, F-13397 Marseille Cedex 20, France.

\section{References}

1. A. Rahmani, P. C. Chaumet, and F. de Fornel, Phys. Rev. A 63, 0238-19 (2001).

2. G. S. Agarwal, Phys. Rev. A 11, 230 (1975).

3. G. S. Agarwal, Phys. Rev. A 12, 1475 (1975).

4. E. M. Purcell and C. R. Pennypacker, Astrophys. J. 186, 705 (1973).

5. B. T. Draine, Astrophys. J. 333, 848 (1988).

6. B. T. Draine and P. J. Flatau, J. Opt. Soc. Am. A 11, 1491 (1994), and references therein.

7. P. C. Chaumet, A. Rahmani, F. de Fornel, and J.-P. Dufour, Phys. Rev. B 58, 2310 (1998).

8. P. C. Chaumet and M. Nieto-Vesperinas, Phys. Rev. B 61, 141-19 (2000).

9. P. C. Chaumet and M. Nieto-Vesperinas, Phys. Rev. B 62, 111-85 (2000).

10. P. C. Chaumet and M. Nieto-Vesperinas, Opt. Lett. 25, 1065 (2000).

11. P. C. Chaumet and M. Nieto-Vesperinas, Phys. Rev. B 64, 0354-22 (2001)

12. A. Liu, A. Rahmani, G. W. Bryant, L. Richter, and S. Stranick, J. Opt. Soc. Am. A 18, 704 (2001).

13. A. Rahmani and G. W. Bryant, "Spontaneous emission in microcavity electrodynamics," Phys. Rev. A (to be published).

14. A. Rahmani and G. W. Bryant, Phys. Status Solidi B 224, 807 (2001).

15. H. Chew, J. Chem. Phys. 87, 1355 (1987).

16. H. Chew, Phys. Rev. A 38, 3410 (1988).

17. B. R. A. Nijboer and F. W. De Wette, Physica 24, 422 (1958).

18. A. Rahmani and G. W. Bryant, Opt. Lett. 25, 433 (2000).

19. F. W. De Wette and G. E. Schacher, Phys. Rev. 137, A78 (1965). 